\title{
Health care challenges at mass gatherings
}

\author{
Athanasios Zafeirakis ${ }^{1}$, Panagiotis Efstathiou ${ }^{2}$ \\ ${ }^{I}$ Nuclear Medicine Department, Army Share Fund Hospital, Athens, Greece \\ ${ }^{2}$ National Health Operations Centre of the Hellenic Ministry of Health, Athens, Greece
}

Received: 2020-07-29.

Accepted: $2020-08-18$

This work is licensed under a Creative Commons Attribution 4.0 International License

\section{J Clin Med Kaz 2020; 6(60):23-28}

Corresponding author: Athanasios Zafeirakis.

E-mail: athzafeirakis@gmail.com, a.g.zafeirakis@army.gr; ORCID:

\begin{abstract}
Mass gatherings are unique events carrying the potential to severely strain public health planning and infrastructures by triggering the entire emergency mechanism of a community or nation. That is mostly the case in terrorist attacks, an overwhelming, though not the most common health-related hazard in mass gatherings. The implementation of a protective mechanism against such threats is pronouncedly cumbersome and requires a complex administrative strategy, detailed operational planning and also a deep interoperability of the major health and homeland security stakeholders. That can be accomplished with an Incident Command System that affords organizational guidelines and flexible tactical planning, which refer to actions undertaken before, during and after the incident. The main challenges before the health incident are named preparedness, surveillance, incident action plans, environmental health/food safety and personnel training; the objectives to be achieved during the main phase of the incident are communication, response, dispensing planning and interoperability. Of the post-incident phase, the most important objectives are the mental first aid provision and the transparent key-messages of post hazard communication.
\end{abstract}

Key words: mass gatherings, public health, incident command system

\section{Introduction}

Mass gatherings (MGs) are defined as organized or spontaneous concentrations of a great number of people with common features (political, social, religious, sports, etc.) which bring them together at a specific (relatively confined) location and for a defined (mostly short) period of time [1,2]. MGs are divided into planned and spontaneous ones. The planned MGs regard two types of events; single, e.g. opening celebrations and recurrent events, such as local festivals, football cups, etc. The spontaneous mass gatherings regard either the same location or unpredicted sites, such as protests, etc [3].

Perhaps the most pronounced characteristic of MGs is that they carry the potential to severely strain or overwhelm the routine function of health infrastructures of the hosting community to such a degree that requires the activation of the emergency mechanism of public health services or/and of civil protection [4]. The most common health-related hazards of MGs are the following $[5,6]$ :
1. Hygiene disorders
2. Temperature and dehydration disorders
3. Infection diseases
4. Accidents and crash injuries
5. Panic reactions

\section{Alcohol and drug abuse}

7. Terrorist attacks

Concerning in particular the terrorist attacks in MGs, the major health care challenges are generally the following: Attacks are often sudden, violent and involve multiple hits overflowing and disorganizing existing health facilities, with massive casualties and critical injuries to an enormous number of victims; radiological, chemical or biological threats are also probable (or speculated), which further enhance public anxiety. Moreover, they provoke a widespread of panic, mostly through social media and -even worse- often in global live broadcast. As the concept of modern terrorism has nowadays shifted from "the strongest possible psychological impact on masses" to "just as many casualties as possible" [7], the risks of otherwise rather insignificant health threats can be thus enormously enhanced [8].

As regards the specific category of religious MGs contagious diseases are still the main health hazard [9]; however these crowding events also constitute an attractive target for terrorists because of some specific reasons [10]:

1. Religious MGs attract a large number and high proportion of aged and of reduced mobility population, thus prerequisite both higher levels of health services and 
accurate on-site medical first aid facilities.

2. The single occurrence and limited duration of the religious events is characterized by deficient preparatory measures by the organizers and by inadequate emergency medical infrastructures.

3. The high density and standing status of the participating population increases the risk, probability and absolute number of casualties in the scenario of a terrorist attack.

4. The inherently highlighted profile of religious gatherings draws more easily adverse reactions from fundamentalists and radicalized opponents.

5. Finally, the relatively homogenous population in terms of nationality and religion further decreases the perceived vulnerability of the participants and enhances a dangerously deceptive sense of crowd security.

The implementation of a protective mechanism against those threats is pronouncedly cumbersome and requires a complex administrative strategy, a detailed operational planning and also a deep interoperability of the major health and homeland security stakeholders [11].

\section{Discussion \\ Incident Command System}

According to the glossary of WHO, as Incident Command System (ICS) is defined "a direction and control scheme used by first responders and other agencies to manage emergencies" [3]. A more detailed definition would describe it as the standardized application of administration, roles, functions and duties by virtue of an explicit authority, based on various operation protocols for each specific emergency. The ICS affords organizational guidelines and flexible tactical planning, known as Incident Action Plans (IAPs). These are brief or extended, according to the short- or long-term character of emergency incidents and describe (often in an escalation process) how actions should be taken and who is responsible for taking those. In the case of MGs IAPs refer to actions undertaken before, during and after the incident; these will be bellow analyzed.

\section{A. Before the incident}

The major involved stakeholders are the organizers of the MG event, the low enforcement authorities and public health agencies. These should consider the type of the event, the medical needs and the safety of the participants [3]. The main challenges before the health incident are the following:

1. Preparedness

2. Surveillance

3. Incident Action Plans

4. Environmental health \& Food safety

5. Personnel Training

\section{Preparedness}

The term refers to the development of a multi-level risk assessment and response planning for all potential health hazards, along with the development of appropriate national stockpiles of resources to support health operations at national level during a MG emergency [12]. The preparedness principle aims at the following objectives:

1. Analysis of former operations and incidents.

2. Updating of existing disaster planning and memoranda of action.

3. Equipment sufficiency and renewal.

4. Legal authority and jurisdictional framework.

5. Infectious diseases vigilance.
6. Consideration of the possibility of the deliberate use of explosives, biological and chemical agents or radioactive materials.

7. Cooperation with all relevant stakeholders (international and public health officials, emergency first-responders, and primary health care providers).

The equipment implied in the above planning includes high-technology infrastructure as well as all the necessary means and procedures to support an efficient and weatherproof communication, management of available data and a secure and real time information flow to multiple receivers. Moreover, the provision of emergency medical care at MGs is uniquely challenging because adequate crowd security needs to be provided; planning should take into account crowd size and factors such as type of event and evacuation plans [13]. According to the conceptual model proposed by Arbon [14] and data from previous review studies [15,16], MG health care planning should take into account three major domains, regarding both individuals and crowds:

1. The psychosocial, which considers the size of the crowd, motivation, mood, behavior, interests, culture, length of stay and abuse of alcohol or drugs.

2. The environmental, which considers the density and mobility status (seated or standing) of the crowd, type of the event, venue characteristics (indoor/outdoor, bounded/ unbounded, focused/extended) weather, temperature and humidity.

3. The bio-medical, which considers the average age, sex, health status, activity level, pharmaceutical and temperaturerelated physiology.

Services that should participate in preparedness planning for mass gatherings include the following [17]:

1. Emergency medical on-site field teams

2. Patient transportation ambulances and paramedical services

3. Emergency departments of Hospitals

4. Mortuary facilities

5. Nursing services

6. Infection control organizations

7. Blood transfusion services

8. Decontamination teams

9. Logistics

10. Volunteer organizations

\section{Surveillance}

The novel concept of an advanced continuous observation and reporting system is of utmost importance for optimal surveillance and investigation of communicable diseases and noninfection threats during MGs [18]. Radiation exposure and toxic surveillance can be accomplished via radiation survey meters and poison control or drug information centers respectively, where they exist; as regards the deliberate use of biological agents or an epidemic outbreak, the syndromic surveillance consists in data collection for symptoms (syndromes) rather than confirmed diagnosis, in contrast to traditional disease surveillance systems which collect data for diagnosed cases of disease. Furthermore, a sophisticated system collects and interchanges specific vital information for all potentially vulnerable civil areas by making use of a broad visual surveillance network that monitors possible threats according to certain hazard thresholds. Subsequently follows the electronic processing of the whole dataset from an interactive mapping application [19]. In all cases, an accomplished real-time collection of data regarding natural or 
deliberate use of biological, chemical or radiological threats leads to a more timely recognition of health hazards, thus enhancing the ability of public health to respond and minimize the impact of such events.

\section{Incident action plans}

Operational planning comprises preparedness and response activities provided from trained personnel capable of prompt responding after identification of a major health incident. Specific objectives in mass incidents include [20,21]:

1. Risk assessment: What might happen and how likely is it to happen?

2. Response: What will we do when it happens?

3. Medical system resilience: The ability of a healthcare system to recover after a disastrous incident.

4. Responders' safety: The protection of healthcare responders during hazardous operations by use of protective clothes, equipment, vaccines, etc.

5. Information management: The collection, analysis and evaluation of all relevant data.

6. Logistic support: The full range of financial and administrative support of health operations regarding medical supplies, general resources and food provision.

7. Time management: The addressing of time-sensitive health emergencies on a rapid medical intervention so as to minimize the number of casualties.

\section{Environmental health \& food safety}

Environmental health and food safety for MGs include the following objectives: Food and drinkable water preparation, hygiene and service, quality of air, sanitation and waste disposal, accommodating facilities, travel sites and possible causes of health incidents (weather, wind, temperature, buildings, etc). Public health services inspect on site, select samples for laboratory testing and monitor problems or law violations by MGs organizers [22].

\section{Personnel training}

The main scope of personnel training is based on a realistic, constant and competent preparatory education along with onfield practice of all the staff involved in emergencies, according to relative official national and international guidelines. Furthermore, such training includes operational planning for future emergency response scenarios, regular reviewing of existing rules and policies and most of all, periodically organized full-scale realistic exercises [23]. The levels of casualty incident training for emergency health responders range from the basic training (recommended for all first responders), to intermediate training (for responders who assume medium-level operational roles, such as field leaders, staff of the National Public Health Organization, National Center for Emergency Assistance, etc.), up to senior management training (recommended for senior leaders, managers and directors, who will mobilize resources in national or international level and will interact with the mass media). The final goal of personnel training is an overall enhancement of alertness and competency, along with continuous updating of operational procedures.

\section{B. During the incident}

Objectives to be achieved in the main operational phase of health functions are the following:

1. Communication

2. Response
3. Dispensing planning

4. Interoperability

\section{Communication}

The communication amidst health crises is a challenging practice with some special tactical characteristics; it typically functions on an emergency basis, in a limited time span, regards sudden and shattering events, often uses unconventional or even old-fashioned media (land lines, CB-radios and walkie-talkies), usually lacks vital data and is, at least initially, frequently dubious. In the initial stages the communication relies on incident recognition, hazard analysis and prompt involvement of relevant stakeholders, preferably in multi-lingual approach. In the course of the crisis the communication system gathers solid and continuous information, analyzes data and influences positively the public opinion by explaining and persuading [24]. The key for a successful communication during the health crisis event is the sum of credibility and trust; credibility results from information accuracy, rapid transmission, clear instructions and common language/nomenclature; trust comes from empathy and honesty. The information flow should be realistic, balanced, integrated, transparent, undogmatic, reassuring and prioritizing. Furthermore, confidentiality of sensitive personal data and protection of human privacy and dignity are necessary for a proper health crisis communication system. Finally, the speed of information transmission usually functions against accuracy; unfortunately that excessive amount of frequently inaccurate information ("infodemic") can easily tangle facts with fiction.

\section{Response}

The term is referred to the effectiveness of the health sector in disaster events. The plan addressing the health system response to a mass-casualty incident should be a cooperative effort between the local, national and international inter-operating stakeholders (analyzed bellow). That is accomplished in the first place with field-level rapid deployment medical forces [25], called Trauma and Critical Care Teams (TCCTs), or Disaster Medical Assistance Teams (DMATs) or in extreme cases even by a rapid set up of field hospitals. The rescue objective of these teams is to provide the best possible health care to victims by the following Standard Operating Procedures (SOPs):

1. Rapid decisions.

2. Multiple, compact emergency medical teams.

3. Simple and standardized triage and first aid plan.

4. Appropriate distribution of emergency medical supplies.

5. Security of operating personnel.

6. Preparedness of satellite Hospitals to address the overflow of victims, along with activation of the supplementary emergency personnel pool. It is estimated that within the first ninety minutes following a major health incident $50-80 \%$ of casualties will arrive at the nearest hospital, albeit that percentile can be affected by many factors, like geospatial location, transport conditions, collateral side effects of the disaster, security issues, etc.

7. In the disaster zone the rescue teams make triage by using the four-colored card emergency standardization, which corresponds to the respective clinical status of the victims. This illustration shows a brief medical history and the degree of medical priority for each victim [26]. Thus, at the point of disaster the TCCTs/DMATs should undertake the following SOPs:

a. Fast, on field estimation of hazards and of probable 
number of casualties.

b. Notification about urgent requirements in personnel and specific rescue and life support equipment (ambulances, bedding, paramedics, body bags for fatalities, cold storage facilities, drinking water supply, etc).

c. Direct delineation of the disaster area for emergency health operations.

d. Outside that area the TCCT/DMATs may support the needs of milder injuries.

\section{Dispensing planning}

After the initial triage each health casualty should evacuate the area of the disaster, following one of the two casualtiesdispensing schools [27]; in the first, the casualties are urgently transported to the nearest advanced health care facility as fast as possible so that precious time to be gained even to the cost of a less comprehensive initial medical care ("scoop and run" principle); in the second school the casualties are not removed until full first-line medical treatment is applied, so that comprehensive medical care to be offered on the field of disaster (and simultaneously decongesting secondary stations), but to the cost of prompt transportation to base hospitals ("stay and play" principle). Of course intermediate protocols also exist. In action a dispensing plan deals with the following problems:

a. Multiple emergency incidents (often in multiple locations) might well occur and thus can easily overwhelm the operational capacity (response to multiple incidents) and capability (coping with unusual or special medical requirements) of the local emergency first aid stations [28].

b. The above situation can gravely confound health personnel, especially when taking into account that there may be severe shortage of specialized staff to cope with the demands of the health incident and also that those personnel should often set triage priorities very hastily, after having been operating without rest for many hours.

c. Defective (if any) patient database is often available in mass disasters; that is mostly the case in MGs, with the notable exception of events where nominal tickets are available.

d. Massive pressure of enormously many, not seriously (or not really) injured, but overwhelmingly concerned or panicked people, who interfere with health responders might also lead the medical system to collapse.

\section{Interoperability}

The terms emphasizes in coordination of national agencies that cover all sorts of planned or spontaneous MGs with any potential kind of health threat in (or coming from) land, sea and air. Most agencies are under the operational control (yet in fewer cases under the command) of the General Secretariat for Civil Protection, GSCP). The long list of those agencies includes, but is not limited to the following: Fire Brigade; Police; Ministry of National Defence; Coast Guard; Ministry of Transport and Communications; Civil Airlines; Medical School Laboratories; National Public Health Organization; National Center for Emergency Assistance (NCEA); Hospital units (public and private); National Atomic Energy Committee; Forensic Services; Ministry of Foreign Affairs; Non-governmental healthcare and humanitarian responders [22]. Of those inter-operating partners perhaps the most important function regards the coordination of satellite hospitals; regional to the affected area hospital units must be able to interchange patients and resources under central coordination. Especially when the disaster exceeds the capacity/ capability of the nation's health system and specialized personnel or equipment is needed, then international mobilization should be requested by making a timely request to the European-CDC, or to the Monitoring and Information Center (MIC), depending on the nature of the incident. Finally, primary (communitybased) health care can be provided to minor injuries by sentinel health units after initial triage and dispensing from the disaster area.

\section{Following the incident}

The post-incident phase functions as a period of relaxation for strained personnel and also as a time of critical reassessment of implicated operational plans. Apart from longer-term evaluation, there are some medium-term objectives to be achieved during return of the health mechanism to ordinary regularity:

1. Hospitalization of severe cases.

2. Physical rehabilitation of victims (for psychological aid see below).

3. The fatality management team affords technical support for casualties, documents human remains, collects and evaluates fingerprints, DNA and dental samples for victim identification and forensic medicine purposes.

4. Preparedness for new incidents (e.g. another terrorist attack soon after the previous) or relapse of former health incidents (e.g. reemergence of a thought-to-be-controlled communicable disease). For that objective to be achieved, predictive epidemiological algorithms are needed, by using multi-level information and international collaboration for the exchange of expertise and experience.

5. Self-evaluation of the health operation system both as a whole and as sub-functions; that crucial procedure yields conclusions and planning ideas for improved future operational doctrines.

Apart from the above synoptic objectives, there are two more that need to be deeper analyzed:

\section{a. Mental first aid provision}

The term "Mental Health First Aid" defines a humane supportive practice in people who had been exposed to recent, acute psycho-pressing factors. Mental health teams, staffed with psychiatrists, psychologists and social workers evaluate early post-disaster stress syndromes of the affected population and provide psychological support by pharmacological and nonpharmacological interventions, in collaboration with religion representatives as well as by maintaining operational call centers (preferably multi-lingual) for public information till well after the ending period. The whole process also includes long-term planning for post-incident mental health support for victims and vulnerable populations and also recognition of corresponding psycho-clinical syndromes in rescue staff, known as "posttraumatic stress syndromes" [29] and "compassion fatigue" [30]. Furthermore, the Victim Information Team, by making use of exclusive call centers $24 \mathrm{~h} / 7 \mathrm{~d}$, acts as liaison between the afflicted population and state authorities to serve their needs and to provide discreet and detailed information regarding the list of missing persons.

\section{b. Post hazard communication}

The community is especially sensitized directly following a disastrous incident; that fact offers the potential for a better risk prevention and mitigation education immediately after an emergency incident. The communication phase of post event 
period aims at the preservation of public composure along with avoidance of panic dissemination and includes the following objectives [22]:

a. Sending a clear, correct and honest message of the State about the incident, i.e. how things went wrong and what rescue and reparatory actions are undertaken and ongoing. The key message should be adequately explanatory, describing what, where, how happened, what should the public do and expect from now on and where they can find answers and get more information. That is especially important when the acute phase of the incident subsides and for many people there is now time for more complex and painful questions.

b. Managing (preferably optimistically) public expectations for what they should wait thence for. That is vital in complicated mass disasters, wherein prompt and proper response is not always easily achievable. Furthermore, such a policy hampers the dissemination of misinformation and rumors through social media.

c. And finally, declaring what lessons were learnt after elaborate analysis of all collected data (mostly from forensic research) and what precautionary measures will be undertaken so that such situations to be avoided in the future. And if possible, who is responsible and for what; that is also essential when the acute phase of the incident will be replaced by anger and desire for justice administration.

\section{Conclusion}

For an optimal and accurate coverage of health incidents in mass gatherings, increased awareness is required for the development of accurate prevention, management and control procedures and infrastructures to enforce the emergency sector of the health system, so that before, during and right after natural and man-made disasters, the health impact to be mitigated and the population to be protected as much as possible. Perhaps the key-message regarding management of health care challenges at mass gatherings is that of a better preparedness on behalf of the national and international authorities, combined with adequate response planning, investments and corresponding public training.

Disclosures: There is no conflict of interest for all authors.

\section{Acknowledgements: None}

Funding: None

\section{References}

1. Turris SA Lund A and Bowles RR (2014) An analysis of mass casualty incidents in the setting of mass gatherings and special events. Disaster Med Public Health Prep. 2014; 8:143-149. DOI: 10.1017/dmp.2014.24.

2. Aitsi-Selmi A, Murray V, Heymann D, McCloskey B, Azhar EI, Petersen E, et al. Reducing risks to health and wellbeing from mass gatherings: the role of the Sendai Framework for Disaster Risk Reduction. Int J Infect Dis. 2016; 47:101-104. DOI: 10.1016/j. ijid.2016.04.006.

3. Endericks T. Public health for mass gatherings: key considerations. WHO 2015. ISBN 978920694385.

4. Locoh-Donou S, Guofen Y, Welcher M, Berry T, O'Connor RE and Brady WJ. Mass-gathering medicine: a descriptive analysis of a range of mass-gathering event types. Am J Emerg Med. 2013; 31:843-846. DOI: 10.1016/j.ajem.2013.01.016.

5. Milsten AM, Maguire BJ, Bissell RA and Seaman KG. Mass gathering medical care: A review of the literature. Prehosp Disast Med. 2002; 17(3):151-162. DOI: 10.1017/s1049023x00000388

6. Memish ZA, Steffen R, White P, Dar O, Azhar EI, Sharma A, et al. Mass gatherings medicine: public health issues arising from mass gathering religious and sporting events. Lancet. 2019; 393:2073-2084. DOI: 10.1016/S0140-6736(19)30501-X.

7. Art RJ. A Grand Strategy for America. Cornell University Press Publishing 2003; 16-18.

8. Karami M, Doosti-Irani A, Ardalan A, Gohari-Ensaf F, Berangi Z, Massad E, et al. Public Health Threats in Mass Gatherings: A Systematic Review. Disaster Med Public Health Prep. 2019; 13(5-6):1035-1046. DOI: 10.1017/dmp.2018.161.

9. World Health Organization: Annual review of diseases prioritized under the Research and Development Blueprint. WHO 2018. Meeting report; February 6-7, Geneva, Switzerland.

10. Jaslow D, Yancy A and Milsten A. Mass Gathering Medical Care. Prehospital Emergency Care; 2000; 4(4):359-360. DOI: 10.1080/10903120090941119.

11. Yezli S and Alotaibi B. Mass gatherings and mass gatherings health Saudi Med J. 2016; 37(7):729-730. DOI: 10.15537/smj.2016.7.15419.

12. Lund A, Gutman SJ and Turris SA. Mass gathering medicine: a practical means of enhancing disaster preparedness in Canada. CJEM. 2011; 13:231-236. DOI: 10.2310/8000.2011.110305.

13. Dong YH, Liu F, Liu Y-M, Jiang X-R and Zhao Z-X. Emergency preparedness for mass gatherings: Lessons of " $12 \cdot 31$ " stampede in Shanghai Bund. Chin J Traumatol. 2017; 20:240-42. DOI: 10.1016/j.cjtee.2016.08.005.

14. Arbon P. The development of conceptual models for mass-gathering health. Prehospital and Disaster Medicine. 2004; 19:208-212. DOI: $10.1017 / \mathrm{s} 1049023 \times 00001795$.

15. De Lorenzo RA. Mass gathering medicine: A review. Prehosp Disast Med. 1997; 12(1):68-72. DOI: 10.1017/s1049023x00037250.

16. Zeitz KM, Zeitz CJ and Arbon P. Forecasting medical workloads at mass gathering events: Predictive models as an adjunct to retrospective review. Prehosp Disast Med. 2005; 20(3):164-168. DOI: 10.1017/s1049023x00002399.

17. World Health Organization: Communicable disease alert and response for mass gatherings: Key considerations. WHO June 2008.

18. Elachola H, Gozzer E, Zhuo J, Sow S, Kattan RF, Samara A, et al. Mass gatherings: a one-stop opportunity to complement global disease surveillance. J Health Specialties. 2016; 4:178-85.

19. Castillo-Salgado C. Trends and directions of global public health surveillance. Epidemiol Rev. 2010; 32:93-109. DOI: 10.1093/epirev/ mxq008.

20. World Health Organization: Emergency response framework 2nd ed. WHO 2017; ISBN 978-92-4-151229-9.

21. World Health Organization: Handbook for Developing a Public Health Emergency Operations Centre (PHEOC) Part A: Policies, Plans and Procedures. WHO 2018; ISBN 978-92-4-151512-2. 
22. Tsouros AD and Efstathiou PA. Mass Gatherings and Public Health: The Experience of the Athens 2004 Olympic Games. WHO 2007; ISBN: 9789289072885.

23. World Health Organization: Handbook for developing a Public Health Emergency Operations Centre Part C: Training and Exercises. WHO 2018; ISBN 978-92-4-151513-9.

24. Efstathiou P, Papafragkaki D, Gogosis K and Manwlidou Z (2009). Crisis management in the Health Sector; Qualities and characteristics of health crisis managers. International Journal of Caring Sciences. 2009; 3:105-107.

25. Balajee SA, Pasi OG, Etoundi AGM, Rzeszotarski P, Do TT, Hennessee I, et al. Sustainable Model for Public Health Operations Centers for Global Settings. Emerging Infectious Diseases. 2017; 23:190-195. DOI: 10.3201/eid2313.170435.

26. Sasser SM, Hunt RC, Faul M, Sugerman D, Pearson WS, Dulski T, et al. Guidelines for Field Triage of Injured Patients. MMWR Recomm Rep. 2012; 13(61):1-20.

27. Salhanick SD, Sheahan W and Bazarian JJ. Use and analysis of field triage criteria for mass gatherings. Prehosp Disast Med. 2003; 18(4):347-352. DOI: 10.1017/s1049023x00001308.

28. Barbera J and Macintyre A. Medical Surge Capacity and Capability: A Management System for Integrating Medical and Health Resources During Large-Scale Emergencies. 2nd ed. 2007; CNA Corporation: Institute for Public Research.

29. Carlier IV, Lamberts R, Van Uchelen A and Gersons BP. Disaster-related post-traumatic stress in police officers: A field study of the impact of debriefing. Stress Medicine. 1998; 14(3):143-8.

30. Bellolio MF, Cabrera D, Sadosty AT, Hess EP, Campbell RL, Lohse CM, et al. Compassion fatigue is similar in emergency medicine residents compared to other medical and surgical specialties. Western Journal of Emergency Medicine, 2014; 15(6):629-635. DOI: 10.5811/westjem.2014.5.21624. DOI: 10.1002/(SICI)1099-1700(199807)14:3<143::AID-SMI770>3.0.CO;2-S 